\title{
Regionale Innovationspotentiale in Südostasien \\ Empirische Ergebnisse aus Singapur, Penang (Malaysia) und Thailand
}

\section{Matthias Kiese, Hannover, Javier Revilla Diez, Kiel}

\section{Hintergrund und Fragestellung}

Die südostasiatischen Schwellenländer konnten in den vergangenen drei Jahrzehnten einen historisch einmaligen wirtschaftlichen Aufholprozeß gegenüber den etablierten Industrieländern realisieren. Zwischen 1971 und 1996 wuchs das reale Bruttosozialprodukt in den meisten Ländern dieser Wachstumsregion mit jahresdurchschnittlichen Raten zwischen sechs und zehn Prozent (vgl. SchäTzl 2000: 234 f.). Nach der Asienkrise von 1997/98 kehrten die meisten der aufstrebenden Volkswirtschaften der Region zunächst schnell wieder auf den Wachstumspfad zurück. Dennoch bleiben Zweifel an der Nachhaltigkeit des "Asian Miracle» (Weltbank 1993) zurück, das Krugman (1994) wegen des in ökonometrischen Studien nachgewiesenen geringen Beitrags des technischen Fortschritts als «Mythos» bezeichnete.

Obwohl der technische Fortschritt als eine entscheidende Determinante zur Beurteilung der Zukunftsperspektiven der südostasiatischen Schwellenländer gilt, liegen repräsentative empirische Erhebungen zur Verbreitung von Innovations- und Kooperationsaktivitäten auf der Mikro-Ebene von Unternehmen in Südostasien bislang nicht vor. Während ökonometrische Studien mit einer Reihe methodischer und inhaltlicher Probleme behaftet sind, können Fallstudien allenfalls exemplarische Evidenz liefern. In einigen Ländern Südostasiens werden von staatlicher Seite periodische Erhebungen der Forschungsund Entwicklungsaktivitäten durchgeführt, beispielsweise seit 1978 in Singapur (jährlich seit 1990, zuletzt AGENCY for Science, Technology and Research 2003) oder seit 1992 alle zwei Jahre in Malaysia (zuletzt MaLAYSIAN SCIENCE AND TeChnology Information Centre 2002). Diese Erhebungen vermögen jedoch nur die Spitze des Eisbergs der Innovationsaktivitäten in Entwicklungs- und Schwellenländern zu erfassen. Wie WoNG (1995:2) betont, kommt der Generierung technischen Wissens mittels Forschung und Entwicklung $(F \& E)$ in Schwellenländern eine geringere Bedeutung zu als in Industrieländern; von größerer Relevanz sei dagegen die Diffusion von importiertem Wissen durch Adoption und Adaption. Auf F\&E beschränkte Erhebungen greifen demnach in diesen Ländern zu kurz.

Um in einem zunehmend internationalen und wissensintensiven Standortwettbewerb ein hohes Einkom- mens- und Beschäftigungsniveau sichern zu können, müssen räumliche Wirtschaftssysteme unterschiedlicher Maßstabsebene (Standorte, Regionen, Nationen) kontinuierlich neues Wissen hervorbringen oder anwenden, wie es vor allem innovative Produkte und Fertigungsverfahren verkörpern. In welchem Ausmaß dies gelingt, hängt in erster Linie von der regionalen Ausstattung mit Innovationsakteuren ab, insbesondere mit innovierenden Unternehmen des Verarbeitenden Gewerbes, wissensintensiven Dienstleistern (Knowledge-Intensive Business Services, KIBS) sowie Forschungseinrichtungen. Interaktive Innovationsmodelle wie das von Kline \& Rosenberg (1986) oder auch Hayeks Konzept der Wissensteilung (HAYEK 1976) implizieren, daß die Innovationsfähigkeit einer Region außerdem maßgeblich von den Interaktionen der Innovationsakteure sowie ihrem weiteren Handlungsumfeld beeinflußt wird. Dieser Erkenntnis tragen die Konzepte territorialer (nationaler, regionaler) Innovationssysteme Rechnung: Das Konzept der nationalen Innovationssysteme wurde unabhängig voneinander von Freeman (1987), Lundvall (1992) und Nelson (1993) eingeführt, während der Begriff der regionalen Innovationssysteme auf COоKE (1992) zurückgeht. Da neues Wissen zunächst noch unvollständig kodifiziert ist («implizites Wissen», Polanyı 1985), wird dessen Austausch durch die face-to-face-Kontakte ermöglichende räumliche Nähe der Innovationsakteure begünstigt. Dieser Aspekt bildet einen zentralen Bestandteil der geographischen Innovationsforschung, wo er empirisch in der spillover-Forschung oder theoretisch in den Konzepten der regionalen Innovationssysteme sowie der innovativen bzw. kreativen Milieus (vgl. SCHÄTZL 2003: 231 f.; Koschatzky 2001, Kap. 5) Ausdruck findet.

Neben den neuen Erkenntnissen der geographischen Innovationsforschung sind weitere Aspekte im Entwicklunsgprozeß Südostasiens zu beachten:

1. Nachholende Industrialisierung: Die späte Industrialisierung bietet den Schwellenländern Südostasiens die Möglichkeit, auf die neuesten verfügbaren Technologien der Industrieländer zurückzugreifen und so ältere Stufen der technologischen Entwicklung zu überspringen. Diesem leapfrogging-Argument steht jedoch die evolutionsökonomische These von der Pfadabhängigkeit der ökonomisch-technischen Entwicklung gegenüber.

2. Dominanz multinationaler Unternehmen (MNU): Adäquate Absorptionskapazitäten vorausgesetzt, kann die hohe Präsenz von MNU südostasiatischen Schwellenländern den Zugriff auf weltweit ver- 
fügbares technisches Wissen ermöglichen. Hiermit sind jedoch auch Gefahren für die Entwicklung endogener technologischer Kompetenzen verbunden.

3. Kulturelle Determinanten: $\mathrm{Zu}$ den Faktoren, die im Rahmen eines Innovationssystems das Innovations- und Kooperationsverhalten der Akteure beeinflussen, zählt nicht zuletzt auch das kulturelle Umfeld.

Vor diesem Hintergrund zielt der vorliegende Beitrag darauf ab, das regionale Innovationspotential sowie die Kooperationsbeziehungen zwischen den Innovationsakteuren in den ausgewählten Untersuchungsregionen Singapur, Penang (Malaysia) und Thailand empirisch zu untersuchen und vergleichend zu bewerten. Zur besseren Einordnung der Untersuchungsregionen sollen auch die im Rahmen des ERIS (European Regional Innovation Survey)-Projekts in elf europäischen Regionen erhobenen Daten in den Vergleich eingebunden werden. Die vorgestellten Analysen sind Ergebnis von Forschungsprojekten, die von der Deutschen Forschungsgemeinschaft (DFG) mit Sachbeihilfen unterstützt wurden («Entwicklung regionaler Innovationspotentiale und innovativer Netzwerke in Südostasien» DFG-Gz.:SCHA 198/38-1) sowie zahlreichen Projekten im Rahmen des DFG-Schwerpunktprogramms «Technologischer Wandel und Regionalentwicklung in Europa» (vgl. SChäTZl \& Revilla Diez 2002).

Die vorgestellten Ergebnisse konzentrieren sich auf das Verarbeitende Gewerbe in Singapur, Penang und Thailand. Nach einer kurzen Einführung in die theoretischen und methodischen Grundlagen des Forschungsprojekts sollen im vierten Abschnitt Ergebnisse aus drei thematischen Schwerpunkten der Untersuchung vertieft diskutiert werden: die technologische Leistungsfähigkeit der Industrieunternehmen in den Untersuchungsregionen, ihre Neigung zu Kooperationen mit externen Partnern im Innovationsprozeß sowie die räumliche Verteilung dieser Kooperationspartner bzw. Reichweiten der Kooperationsbeziehungen. Abschließend werden die wesentlichen Erkenntnisse zusammengefaßt und ungelöste Forschungsfragen aufgeworfen.

\section{Theoretische Grundlagen}

Zentrale Begriffe der neueren Ansätze zur Innovationsforschung sind institutionelle Rahmenbedingungen, kollektives Lernen und Kooperation. Stand zu Beginn noch der Nationalstaat im Vordergrund, kommt der Region eine immer größere Bedeutung zu. Die stärkere Betonung der regionalen und lokalen Ebene hat seit Beginn der 90er Jahre zahlreiche Arbeiten zu regionalen Innovationssystemen entstehen lassen (CoOKe \& Morgan 1998; BraczyK et al.
1998; Maskell et al.1998). Auch hier ist der Ausgangspunkt das interaktive Innovationsmodell, das starke Rückkopplungsprozesse und somit intensive Verflechtungsbeziehungen zwischen unterschiedlichen Akteuren betont. Die Autoren übertragen die systemischen Elemente eines nationalen Innovationssystems auf die regionale Ebene. Kernaussage ist, daß in Regionen spezifische Umfeldbedingungen und Verflechtungsbeziehungen zwischen unterschiedlichen Akteuren anzutreffen sind, die das regionale Innovationsgeschehen beeinflussen und sich positiv oder negativ auf die Ausschöpfung des regionalen Innovationspotentials auswirken. Im Zentrum eines regionalen Innovationssystems stehen folgende Akteure (COOKE \& Morgan 1998):

- Unternehmen des Verarbeitenden Gewerbes:Unternehmen sind zum einen Empfänger neuen Wissens bzw. neuer technologischer Problemlösungen; zum anderen sind sie in der Lage, durch eigene F\&EAnstrengungen neues Wissen zu generieren.

- Unternehmens- bzw. innovationsorientierte Dienstleistungsunternehmen: Auch sie sind sowohl Empfänger als auch Entwickler neuen Wissens. Im Zuge der zunehmenden Flexibilisierung und Spezialisierung der Produktion greifen Unternehmen des Verarbeitenden Gewerbes bei der Realisierung von Produkt-, Prozeß- und/oder organisatorischen Innovationen immer stärker auf spezialisierte Dienstleistungsunternehmen zurück.

- Forschungseinrichtungen: In der Regel aus Mitteln der öffentlichen Hand finanziert, betreiben sie Grundlagen- und angewandte Forschung. Sie sind wesentlich an der Entstehung und Diffusion neuen Wissens beteiligt. Über internationale Forschungskooperationen haben sie Zugang zu international verfügbarem Wissen, das durch enge Zusammenarbeit mit regionalen Akteuren auch zur Lösung regional-/lokalspezifischer Unternehmensprobleme angewendet werden kann («Antennenfunktion», vgl. Revilla Diez 2000: 459-461).

Die Umfeldbedingungen werden durch folgende Elemente beeinflußt:

- Innovations- und diffusionsunterstützende Einrichtungen des privaten und öffentlichen Sektors: Unterschiedliche Einrichtungen versuchen, die Entstehung und den Transfer von Wissen zwischen Wissensproduzenten und Anwendern bzw. Nutzern zu verbessern. Hierzu zählen Transfer- und Informationsvermittlungsstellen, Technologie- und Gründerzentren, Kammern, aber auch Finanzinstitutionen wie etwa Banken.

- Marktimpulse: Der Markt ist ein wichtiger Anreizmechanismus für die Realisierung von Produkt-, Prozeß- oder organisatorischen Innovationen. Technologische Neuerungen, z.B. in Form neuer Produkte, gelangen über den Markt an 
die Verbraucher. Bereits in frühen Phasen des Innovationsprozesses kommt es zu Wechselwirkungen zwischen Markt und Entwicklern (vgl. HipPEl 1988).

- Bildungssystem: das Bildungssystem ist verantwortlich für die Verfügbarkeit qualifizierter Arbeitskräfte, die einerseits bei der Wissensentstehung, aber auch bei der Umsetzung in Produkte bzw. Prozesse beteiligt sind.

- Staat bzw. öffentliche Verwaltung: In Abhängigkeit von ihrer direkten Einflußnahme können sie sich positiv auf das Innovationsverhalten in einer Region auswirken, z.B. indem sie die F\&E-Kapazitäten der Unternehmen und der Forschungseinrichtungen stärken sowie die Zusammenarbeit zwischen diesen Akteuren fördern.

- Unternehmenskultur: Die Funktionsfähigkeit eines regionalen Innovationssystems hängt entscheidend davon $\mathrm{ab}$, in welchem Maße die unterschiedlichen Akteure miteinander vernetzt sind und gemeinsame Lösungen anstreben. Das setzt eine Kooperationsneigung voraus, die im Wesentlichen von einer gemeinsamen Vertrauensbasis abhängig ist. Das sozio-kulturelle Umfeld kann institutionelle Regelungen hervorbringen, die zu routinemäßigen Verhaltensmustern und zur Verringerung von Unsicherheiten führen können.

Im Innovationsprozeß können Netzwerke bei der Partnersuche, der gemeinsamen Forschung und Entwicklung und der Diffusion technischer Innovation entscheidend zur Senkung von Transaktionskosten beitragen (Picot 1982; Williamson 1989; Meyer 1995; SCHEIDT 1995). Bestehende Institutionen können dabei die Höhe der Transaktionskostenreduzierung entscheidend beeinflussen (HERDEN 1992). Neben geeigneten formalen Rahmenbedingungen prägen informelle Institutionen (z.B. kulturelle Standards) die Austauschbeziehungen zwischen den Akteuren und erklären somit die unterschiedliche Innovationsfähigkeit und Wirtschaftsleistung von Regionen und Ländern. Räumliche Nähe, gute Kommunikationsverbindungen, ein gemeinsamer kultureller Hintergrund und eine entwickelte Innovationsinfrastruktur können somit einen Katalysator zur Nutzbarmachung des regionalen Innovationspotentials darstellen (TödTling 1994; GrabHer 1993; HåKanson 1987). Das regionale Innovationspotential wird verstanden als die Ausprägung aller Faktoren, die die Innovationsleistung einer Region bestimmen bzw. hemmen. Es wird geprägt durch die Innovationsakteure einer Region, d.h. vornehmlich Unternehmen und Forschungseinrichtungen sowie durch die Nutzung der regionalen Wissens- und Technikbasis und des innovations- und diffusionsunterstützenden Dienstleistungsangebots, $z$. B. von Transfer- und Informationsvermittlungsstellen.
Um Aussagen über das regionale Innovationspotential treffen zu können, muß eine Analyse der genannten Akteure erfolgen, insbesondere der Industrieunternehmen, der unternehmensnahen Dienstleister sowie der Forschungs- und Entwicklungseinrichtungen. Nur so lassen sich Verflechtungsbeziehungen zwischen den Interaktionspartnern feststellen und ggf. durch Intensivierung und Initiierung von intra- und interregionalen Netzwerken regionale Entwicklungsengpässe beseitigen. Eine Konzentration auf Verflechtungsbeziehungen zwischen Wissenschaft und Wirtschaft, wie sie in der Technologietransferdebatte Anfang der 80er Jahre erfolgte, reicht nicht aus, um ein regionales Innovationssystem zu erfassen. Unter Berücksichtigung des nicht-linearen Innovationsmodells erhält die Analyse von innovativen Netzwerken zwischen Unternehmen sowie zwischen Unternehmen und öffentlichen Forschungseinrichtungen besondere Beachtung. Aus Sicht der Innovationsgeographie stellt sich die Frage, welche Bedeutung der räumlichen Nähe der Akteure im Innovationsprozeß zukommt.

\section{Methodische Vorgehensweise}

Das in zwei Phasen zwischen 1995 und 1999 durchgeführte European Regional Innovation Survey (ERIS) stellte einen ersten Versuch dar, regionale Innovationspotentiale sowie die intra- und interregionalen Kooperationsbeziehungen von Innovationsakteuren empirisch zu erfassen und vergleichend zu bewerten. Dabei wurden in elf europäischen Regionen verwertbare Daten von rund 8.600 Innovationsakteuren erhoben, darunter 4.200 Industrieunternehmen, 2.500 wissensintensive Dienstleister und 1.900 Forschungseinrichtungen. Eine Übersicht über die erste Phase dieses Projekts bieten Fritsch, Koschatzky, Schätzl et al. (1998), über den Stand nach Abschluß der zweiten Phase berichtet Sternierg (2000).

Beim Design der Fragebögen für die postalischen Erhebungen in Singapur, Penang und Thailand galt es, maximale Vergleichbarkeit mit der ERIS-Untersuchung und anderen empirischen Studien wie dem Community Innovation Survey der EUROPÄISCHEN KommisSION (2001) oder dem Mannheim Innovation Panel des Zentrums für Europäische Wirtschaftsforschung (u. a. JanZ \& Licht 1999; JANZ, Ebling, GotTSChalK et al. 2001) zu gewährleisten. Andererseits mußte bestimmten Spezifika der Untersuchungsregion sowie den Interessen der lokalen Kooperationspartner Rechnung getragen werden. Die resultierenden Fragebögen stellen somit einen Kompromiß dar, in dem die Kernelemente der ERIS-Befragungen jedoch erhalten werden konnten:

- Allgemeine Informationen: Einleitend wurden allgemeine Betriebsmerkmale wie Alter, Größe (Um- 
satz, Kapitalstock, Beschäftigte), Branche, Besitzverhältnisse, Funktionalstruktur, Exportanteil, Qualifikationsniveau der Beschäftigten usw. abgefragt. In der Analyse können diese Variablen zur Erklärung von Unterschieden im Innovations- und Kooperationsverhalten herangezogen werden.

- Innovationsaktivitäten: Innovierende Unternehmen, die in den letzten drei Jahren ein neues oder substantiell verbessertes Produkt oder ein ebensolches Fertigungsverfahren eingeführt haben, wurden nach Details zu ihrem Innovationsverhalten befragt. Dabei wurden sowohl Inputindikatoren (Personal und Aufwendungen für $F \& E$ bzw. Innovationen) als auch throughput-Indikatoren (z.B. Patente) und output-Indikatoren erfaßt. Als innovativ gilt ein Unternehmen, das mindestens $25 \%$ seines Umsatzes mit neuen oder substantiell verbesserten Produkten erzielt bzw. einen ebensolchen Anteil seines Produktionsvolumens mit neuen oder verbesserten Verfahren erstellt.

- Innovationskooperationen: In diesem zentralen Teil der Befragungen wurden Unternehmen befragt, welche externen Quellen technischen Wissens sie für ihre Innovationsprozesse nutzen, mit welchen externen Partnern sie zusammenarbeiten und wo diese Partner lokalisiert sind. Die wichtigsten Fragestellungen widmen sich hier dem Zusammenhang von Kooperation und Innovationserfolg sowie der Relevanz räumlicher Nähe für die Zusammenarbeit.

Die postalische Befragung im Verarbeitenden Gewerbe Singapurs wurde zwischen September 1999 und Januar 2000 in enger Zusammenarbeit mit dem Centre for Management of Innovation and Technopreneurship (CMIT) an der National University of Singapore und Singapurs einflußreicher Wirtschaftsförderungsagentur, dem Economic Development Board (EDB), durchgeführt. Von 1.869 versandten Fragebögen konnten 374 Rückläufe verwertet werden, womit die angestrebte Rücklaufquote von $20 \%$ erreicht wurde. Erste Ergebnisse wurden von Wong, KieSE, SingH et al. (2003) vorgestellt, eine detailliertere Analyse des Datensatzes sowie des durch Interviews gewonnenen vertiefenden Fallstudienmaterials erfolgt in KIESE (2004).

Das im Sommer 2000 in Zusammenarbeit mit dem Socio-Economic and Environmental Research Institute (SERI) durchgeführte Penang State Innovation Survey basierte auf einer 951 Unternehmen des Verarbeitenden Gewerbes umfassenden Datenbank. Von den 921 zugestellten Fragebögen wurden 192 in verwertbarer Qualität zurückgeschickt, was einer Rücklaufquote von 20,8\% entspricht. Erste Ergebnisse dieser Erhebung wurden der Regierung des Bundesstaates Penang in einem unveröffentlichten Bericht sowie an einem Workshop vorgestellt (SOCIO-ECONOMIC AND ENVIRONMENTAL RESEARCH
Institute \& Universität Hannover 2001; Ong 2001), eine umfassendere Auswertung der Daten sowie weiterer Interviews erfolgt in STRACKE (2003).

Auf Basis der in Singapur und Penang verwendeten Fragebögen führte das Beratungsunternehmen The Brooker Group Public Company Limited im Auftrag der National Science and Technology Development Agency (NSTDA) zwischen Januar und April 2001 die erste landesweite F\&E- und Innovationserhebung in Thailand durch, die von den Autoren wissenschaftlich begleitet wurde. Aus den 13.415 umsatzstärksten thailändischen Unternehmen wurde mit Hilfe einer nach Branchen und Betriebsgrößenklassen geschichteten Zufallsauswahl eine Stichprobe mit 2.166 Elementen gezogen. 1.019 dieser Unternehmen sandten verwertbare Antworten zurück, was einer hervorragenden Rücklaufquote von $47 \%$ entspricht (vgl. VIRASA \& BRIMBLE 2001).

Tab. 1 stellt die in Singapur, Penang und Thailand durchgeführten Industriebefragungen den Erhebungen des European Regional Innovation Survey (ERIS) gegenüber. Es wird deutlich, daß die in Südostasien realisierten Stichproben absolut wie relativ innerhalb der Spannweite der ERIS-Befragungen liegen und diese im Falle Thailands sogar übersteigen.

\section{Ausgewählte Untersuchungsergebnisse}

\subsection{Technologische Leistungsfähigkeit: Aufholprozesse und Disparitäten}

Mit Hilfe von Sekundärstatistiken läßt sich in den 1980er und 1990er Jahren ein rascher technologischer Aufholprozeß Singapurs nachweisen (Tab. 2). Die Bruttoaufwendungen des Stadtstaates für F\&E erreichten 2002 2,19\% des Bruttoinlandsprodukts (BIP), den höchsten Wert innerhalb Südostasiens. Bis 2005 will Singapur die verbleibende Lücke zu den meisten OECD (Organization of Economic Cooperation and Development)-Ländern schließen, deren F\&E-Intensität sich in einem Korridor zwischen $2 \%$ und $3 \%$ bewegt. Durch Ausbau der nationalen F\&E-Infrastruktur und massive Anwerbung ausländischer Wissenschaftler konnte der Stadtstaat seine Forscherdichte zwischen 1980 und 1995 nahezu verfünffachen und mit 2.318 Forschern je Million Einwohner nahe an den Wert Deutschlands (1995: 2.831) herankommen.

In den südostasiatischen Schwellenländern der zweiten Generation läßt eine Verringerung der technologischen Lücke dagegen noch auf sich warten: Nach Angaben des Malaysian Science and Technology INFORMATION CENTRE (2002) wurden in Malaysia 2000 nur $0,5 \%$ des BIP für F\&E aufgewandt, in Thailand 1995 sogar nur 0,13\% (UNESCO 1999). Schenkt man 


\begin{tabular}{|llccc|}
\hline Region & \multicolumn{1}{c}{ Land } & Jahr $^{1)}$ & Rücklauf & Rücklaufquote \\
\hline Baden & Deutschland & 1995 & 430 & $15,8 \%$ \\
Hannover-Braunschweig-Göttingen & Deutschland & 1995 & 372 & $20,6 \%$ \\
Sachsen & Deutschland & 1995 & 1.004 & $16,7 \%$ \\
Elsaß & Frankreich & 1997 & 263 & $15,0 \%$ \\
Barcelona & Spanien & 1997 & 395 & $15,3 \%$ \\
Gironde & Frankreich & 1997 & 101 & $12,7 \%$ \\
Slowenien & Slowenien & 1997 & 416 & $31,2 \%$ \\
Süd-Holland & Niederlande & 1997 & 261 & $13,7 \%$ \\
Süd-Wales & Verein. Kgr. & 1997 & 280 & $17,6 \%$ \\
Stockholm & Schweden & 1997 & 451 & $24,0 \%$ \\
Wien & Österreich & 1997 & 204 & $19,9 \%$ \\
\hline ERIS-11 & & & $\mathbf{4 . 1 7 7}$ & $\mathbf{1 9 , 7 \%}$ \\
\hline Singapur & Singapur & 1999 & 374 & $20,0 \%$ \\
Penang & Malaysia & 2000 & 192 & $20,8 \%$ \\
Thailand & Thailand & 2000 & 1.019 & $47,0 \%$ \\
\hline
\end{tabular}

1) Start der Befragungen

Tab. 1: Projektgeschichte und Rücklauf (nur Verarbeitendes Gewerbe)

Project background and response (only manufacturing sector)

Histoire du projet et rétrospective (uniquement pour les activités de transformation)

Daten: Eigene Erhebungen (vgl. Kap. 3)

den rudimentären Daten Glauben, hat sich der Abstand zu den Industrieländern seit 1980 eher vergrößert. Auch die Forscherdichte lag in Malaysia und Thailand Mitte der 1990er Jahre deutlich unter dem Wert Singapurs.

Während die auf volkswirtschaftlicher Ebene aggregierten Daten eine rasche Verringerung der technologischen Lücke Singapurs gegenüber den führenden Industrienationen belegen, weist der Vergleich der Ergebnisse unserer Erhebungen mit den ERIS-Daten einen deutlichen Rückstand aller südostasiatischen Untersuchungsregionen auf Unternehmensebene aus (Tab. 3). Dieser Rückstand ist am geringsten beim Anteil innovierender Unternehmen: Während in den ERIS-Regionen durchschnittlich $78 \%$ aller Unternehmen die Einführung eines neuen oder substantiell verbesserten Produktes oder Fertigungsverfahrens angaben, waren es in Singapur $39 \%$ und in Penang $42 \%$. Formale F\&E sowie der Schutz geistigen Eigentums durch Patente spielen dagegen eine geringere Rolle. Am größten ist der Rückstand jedoch beim Umsatzanteil neuer Produkte. Während dieser in den ERISRegionen rund $50 \%$ beträgt. liegt er in Singapur und Penang nur bei etwa $12,5 \%$. Rechnet man multinationale Unternehmen heraus, sinkt dieser Wert auf $8 \%$ ab. In Thailand erwirtschaften die Unternehmen sogar nur $4,7 \%$ ihres Umsatzes mit neuen oder signifikant verbesserten Produkten. Der von input-über throughput- zu output-Indikatoren zunehmende technologische Rückstand läßt auf eine im Durchschnitt geringere Effizienz betrieblicher Innovationsanstrengungen in Singapur, Penang und Thailand schließen, die durch interne Managementprobleme oder ein ungünstigeres externes Umfeld bedingt sein kann.

Ein Vergleich der drei südostasiatischen Untersuchungsregionen untereinander zeigt zunächst, daß Penang und Singapur trotz des deutlichen Vorsprungs Singapurs im nationalen Vergleich auf einer ähnlichen technologischen Entwicklungsstufe stehen. Penang, das als "Silicon Island» (u. a. ChIN \& LeE 1999) über einen bedeutenden Cluster multinationaler ElektronikUnternehmen verfügt, hat einen höheren Anteil innovierender Unternehmen, andererseits aber einen etwas geringeren Anteil F\&E betreibender und Patente anmeldender Unternehmen. Thailand weist dagegen bei allen erhobenen Indikatoren deutliche Rückstände gegenüber Singapur und Penang auf. Insgesamt sind diese Erhebungsergebnisse konsistent mit der sekundärstatistischen Evidenz: Singapur hat bereits eine 


\begin{tabular}{|c|c|c|c|c|c|c|}
\hline \multirow[t]{2}{*}{ a) } & \multicolumn{6}{|c|}{ Anteil der Bruttoaufwendungen für $F \& E$ in $\%$ des BIP } \\
\hline & & 1980 & 1985 & 1990 & 1995 & 2000 \\
\hline Singapur & & $0,26^{1)}$ & $0,54^{2)}$ & 0,86 & 1,16 & 1,91 \\
\hline Malaysia & & n.v. & n.v. & $0,40^{3)}$ & $0,24^{4)}$ & 0,50 \\
\hline Thailand & & 0,39 & 0,34 & 0,18 & 0,13 & n.v. \\
\hline 1) 1981 & 2) 1984 & 3) 1992 & 1996 & & & \\
\hline b) & \multicolumn{5}{|c|}{ Forscher je 1 Mio. Einwohner } & \\
\hline & & 1980 & 1985 & 1990 & 1995 & \\
\hline Singapur & & $485^{1)}$ & 908 & 1.426 & 2.318 & \\
\hline Malaysia & & n.v. & $182^{2)}$ & $327^{4)}$ & $93^{6)}$ & \\
\hline Thailand & & n.v. & $105^{3)}$ & $87^{5)}$ & 118 & \\
\hline 1) 1981 & 2) 1983 & 3) 1987 & 1988 & 1989 & 1996 & \\
\hline
\end{tabular}

Tab. 2: Sekundärstatistische Inputindikatoren: Singapur, Malaysia und Thailand im Vergleich Industrial statistical input indicators: Singapore, Penang (Malaysia) and Thailand in comparison Indicateurs industriels: comparaison entre Singapour, la Malaisie et la Thaïlande Daten: UNESCO (Statistical Yearbook 1999); NSTB (National Survey of R\&D in Singapore, div. Jg.)

höhere technologische Entwicklungsstufe erreicht als Malaysia und Thailand, innerhalb Malaysias stellt Penang jedoch eine high-tech-Enklave dar.

\subsection{Innovation als interaktiver Prozeß}

In der Innovationsforschung hat sich seit den 1980er Jahren die Erkenntnis durchgesetzt, daß Innovationsprozesse zumeist nicht linear ablaufen, sondern vielmehr durch ein hohes $\mathrm{Ma} \beta$ an Komplexität und Rückkopplungen in allen Phasen gekennzeichnet sind (u.a. Kline \& Rosenberg 1986; Koschatzky 2001: 44-48). «No business is an island» (HÅKANSON \& SNEHOTA 1997) - diese Kernaussage neuerer Innovationsmodelle findet sich in unseren Erhebungsergebnissen bestätigt: Nahezu alle Unternehmen kooperieren in ihren Innovationsprojekten mit externen Partnern (Tab. 4). Rund $90 \%$ gaben sogar intensive Kooperationsbeziehungen zu mindestens einem Partner an. Es läßt sich feststellen, daß Unternehmen in Singapur, Penang und Thailand im Rahmen ihrer Innovationsprojekte häufiger auf Kooperationen zurückgreifen als ihre europäischen Pendants. Zwei Erklärungsansätze stehen hierfür zur Verfügung: Erstens läßt eine stärkere Kooperationsneigung nach der ressourcenbasierten Theorie der Unternehmung auf eine schlechtere Ausstattung südostasiatischer Unternehmen mit innovationsrelevanten Ressourcen schließen (vgl. Penrose 1959; Pralahad \& Hamel 1990, siehe auch Wong 1999), zweitens reflektiert sie einfach das höhere Maß an externer Kontrolle.
Wie in den ERIS-Regionen sind auch in Südostasien vertikale Kooperationsmuster vorherrschend: Die wichtigsten Partner sind Kunden, gefolgt von Zulieferern und ausländischen Mutterunternehmen. Dies bestätigt die in der Literatur vertretene Auffassung, daß die wichtigsten Kanäle des Technologietransfers in Schwellenländern zwischen den Hauptquartieren von Multinationalen Unternehmen (MNU) und ihren lokalen Zweigniederlassungen sowie zwischen lokalen Zulieferern und deren technologisch fortgeschrittenen Abnehmern (in Industrieländern oder MNU vor Ort) verlaufen. Variationen in der relativen Bedeutung von Forschungseinrichtungen zwischen den Untersuchungsregionen reflektieren i.d.R. deren quantitative und qualitative Präsenz in der Region, die vor allem in Penang noch gering entwickelt ist. Horizontale Kooperationsbeziehungen mit Wettbewerbern oder sonstigen Unternehmen spielen dagegen wie in Europa nur eine untergeordnete Rolle.

\subsection{Raummuster der Kooperationsbeziehungen}

Eine zentrale Untersuchungshypothese widmete sich dem Zusammenhang zwischen Kooperationsneigung und räumlicher Entfernung der Kooperationspartner. Es wird erwartet, daß räumliche Nähe in den Frühphasen des Innovationsprozesses den Austausch des noch unvollständig kodifizierten Wissens fördert. Abb. 1 vergleicht die Kooperationsreichweiten nach Partnern der Industrieunternehmen in Singapur, Penang und den drei metropolitanen ERIS-Regionen Barcelona, Wien 


\begin{tabular}{|c|c|c|c|c|c|c|c|c|c|}
\hline $\begin{array}{c}\text { Innovationsprozeß } \\
\text { Region }\end{array}$ & Land & $\begin{array}{l}\text { Input } \\
\text { F\&E }\end{array}$ & Patente $^{2)}$ & $\begin{array}{r}\text { Through } \\
\text { In } \\
\text { gesamt }\end{array}$ & $\begin{array}{l}\text { put } \\
\text { novieren } \\
\text { Produkt }\end{array}$ & $\begin{array}{l}d^{3)} \\
\text { Prozeß }\end{array}$ & gesamt & $\begin{array}{l}\text { Output } \\
\text { Innovativ } \\
\text { Produkt }\end{array}$ & $\begin{array}{l}4^{4)} \\
\text { t Prozeß }\end{array}$ \\
\hline ERIS total & & $78,4 \%$ & $24,1 \%$ & $78,1 \%$ & $69,5 \%$ & $62,6 \%$ & n.e. & $49,8 \%$ & n.e. \\
\hline ERIS-Maximum & & $88,5 \%$ & $36,0 \%$ & $93,9 \%$ & $79,1 \%$ & $79,3 \%$ & n.e. & $66,0 \%$ & n.e. \\
\hline ERIS-Minimum & & $70,0 \%$ & $10,3 \%$ & $62,0 \%$ & $49,0 \%$ & $45,0 \%$ & n.e. & $32,8 \%$ & n.e. \\
\hline Singapur & Singapur & $29,7 \%$ & $7,8 \%$ & $39,0 \%$ & $30,2 \%$ & $29,4 \%$ & $19,5 \%$ & $12,3 \%$ & $15,7 \%$ \\
\hline - lokale Unternehmen & Singapur & $25,2 \%$ & $5,5 \%$ & $31,2 \%$ & $21,2 \%$ & $22,8 \%$ & $13,5 \%$ & $8,1 \%$ & $10,3 \%$ \\
\hline - MNU & Singapur & $37,2 \%$ & $11,7 \%$ & $52,6 \%$ & $46,0 \%$ & $40,9 \%$ & $29,9 \%$ & $19,7 \%$ & $25,0 \%$ \\
\hline Penang & Malaysia & $26,6 \%$ & $5,8 \%$ & $42,4 \%$ & $34,6 \%$ & $38,7 \%$ & $20,9 \%$ & $12,6 \%$ & $16,2 \%$ \\
\hline - lokale Unternehmen & Malaysia & $23,9 \%$ & $3,7 \%$ & $36,6 \%$ & $28,4 \%$ & $32,1 \%$ & $14,9 \%$ & $8,1 \%$ & $12,7 \%$ \\
\hline - MNU & Malaysia & $32,8 \%$ & $10,3 \%$ & $56,1 \%$ & $49,1 \%$ & $54,4 \%$ & $35,1 \%$ & $22,8 \%$ & $24,6 \%$ \\
\hline Thailand & Thailand & $15,1 \%$ & $2,2 \%^{5)}$ & $17,8 \%$ & $13,9 \%$ & $12,8 \%$ & $7,5 \%$ & $4,7 \%$ & $5,6 \%$ \\
\hline - lokale Unternehmen & Thailand & $16,1 \%$ & $2,1 \%^{5)}$ & $18,8 \%$ & $15,4 \%$ & $13,3 \%$ & $7,6 \%$ & $5,1 \%$ & $6,1 \%$ \\
\hline$-\mathrm{MNU}$ & Thailand & $12,3 \%$ & $2,3 \%{ }^{5)}$ & $14,6 \%$ & $9,6 \%$ & $11,2 \%$ & $6,9 \%$ & $3,5 \%$ & $4,2 \%$ \\
\hline
\end{tabular}

1) Anteil der Forschung und Entwicklung betreibenden Unternehmen

2) Anteil der Unternehmen mit Patentanmeldungen in den letzten drei Jahren

3) Anteil der Unternehmen, die in den letzten drei Jahren neue oder substantiell verbesserte Produkte oder Verfahren einführten

4) Anteil der Unternehmen, deren Anteil neuer Produkte (Prozesse) am Umsatz (Produktionsvolumen) mindestens $25 \%$ beträgt

5) In Thailand wurden nur F\&E betreibende Unternehmen nach ihrer Patentaktivität befragt.

n.e. nicht erhoben

Tab. 3: Innovationsindikatoren: Untersuchungsregionen im Vergleich Innovation indicators: survey areas in comparison

Indicateurs d'innovation: comparaison des régions soumises à l'enquête

Daten: Eigene Erhebungen (vgl. Kap. 3)

und Stockholm. In Thailand wurde die räumliche Verteilung der Kooperationspartner dagegen nicht abgefragt. Das Netzdiagramm unterscheidet zwischen intraregionalen Kooperationen, nationalen Kooperationen, Kooperationen innerhalb der respektiven Integrationsräume (Europa bzw. ASEAN - Association of Southeast Asian Nations) sowie darüber hinausgehenden Verflechtungen. Dabei läßt sich die Fläche der Polygone als Bedeutung der Kooperationspartner interpretieren, während ihre Form auf die räumliche Ausrichtung bzw. die Reichweiten der innovationsrelevanten Kooperationen hinweist.

In den europäischen Regionen läßt sich beobachten, daß die Kooperationsintensität allgemein mit zunehmender Entfernung der Partner abnimmt. Hier konzentrieren sich die Kooperationsbeziehungen zu gleichen Teilen auf die regionalen sowie nationalen
Innovationssysteme, während Kooperationen auf der mondialen Maßstabsebene wenig verbreitet sind. Intraregionale Kooperationen sind besonders für die Zusammenarbeit mit Dienstleistern und Forschungseinrichtungen von Bedeutung, vertikale Kooperationen mit Abnehmern bzw. Zulieferern entlang der Wertschöpfungskette sind dagegen räumlich stärker diversifiziert.

Die Innovationskooperationen von Industrieunternehmen in Singapur und Penang weisen dagegen ein ausgeprägt diskontinuierliches Raummuster auf. $86 \%$ bzw. $68 \%$ aller innovierenden Unternehmen unterhalten intensive Kooperationen mit Partnern außerhalb Südostasiens, aber nur gut $40 \%$ mit Partnern in den benachbarten ASEAN-Staten. Die meisten Unternehmen «überspringen» damit die technologisch weniger entwickelten Nachbarländer und kooperieren direkt 


\begin{tabular}{|l|r|r|r|r|}
\hline & $\begin{array}{r}\text { Singapur } \\
\text { (n=144) } \\
\text { intensiv }\end{array}$ & $\begin{array}{c}\text { Penang } \\
\text { (n=79) } \\
\text { intensiv }\end{array}$ & $\begin{array}{c}\text { Thailand } \\
\text { (n=177) } \\
\text { intensiv }\end{array}$ & $\begin{array}{r}\text { ERIS-3 } \\
\text { (n= 871) } \\
\text { intensiv }\end{array}$ \\
\hline Beliebiger Partner & $92,4 \%$ & $87,3 \%$ & $88,7 \%$ & $79,9 \%$ \\
Kunden, Abnehmer & $67,4 \%$ & $75,9 \%$ & $70,1 \%$ & $55,3 \%$ \\
Zulieferer & $46,5 \%$ & $55,7 \%$ & $60,5 \%$ & $37,7 \%$ \\
Mutterunternehmen, & & & & \\
verbundenes Unternehmen & $58,3 \%$ & $44,3 \%$ & $42,4 \%$ & n.e. \\
Forschungseinrichtungen & $27,1 \%$ & $11,4 \%$ & $21,5 \%$ & $23,1 \%$ \\
Nicht-technische Dienstleister & $13,2 \%$ & $21,5 \%$ & $12,4 \%$ & \} $42,4 \%$ \\
Technische Dienstleister & $27,8 \%$ & $38,0 \%$ & $20,3 \%$ & $17,9 \%$ \\
Wettbewerber & $9,6 \%$ & $13,9 \%$ & $16,9 \%$ & \} $17,9 \%$ \\
Sonstige Unternehmen & $12,5 \%$ & $6,3 \%$ & $10,7 \%$ & $1 \%$ \\
\hline
\end{tabular}

ERIS-3 Metropolitane Innovationssysteme Barcelona, Wien, Stockholm n.e. nicht erhoben

Tab. 4: Häufigkeit intensiver innovationsorientierter Kooperationsbeziehungen im Verarbeitenden Gewerbe nach Untersuchungsregionen und Partnern

Distribution of intensive innovation-oriented co-operation between manufacturing industries according to survey area and partner

Fréquence des relations de coopération en matière d'innovation dans les activités de transformation, par région et partenaires soumis à l'investigation

Daten: Eigene Erhebungen (vgl. Kap. 3)

mit Partnern in den führenden Technologieregionen in Nordamerika, Europa und Japan. In Singapur wie in Penang läßt sich beobachten, daß MNU aufgrund ihrer Konzerneinbettung und Absatzmärkte stark auf mondiale Kooperationen ausgerichtet sind. Für lokale Unternehmen sind dagegen Kunden in der Region der wichtigste Kooperationspartner - mutmaßlich also MNU-Tochterunternehmen in Singapur und Penang. Nachfolgend sollen die Unterschiede in den räumlichen Kooperationsmustern zwischen den beiden südostasiatischen Untersuchungsregionen herausgearbeitet werden.

In Singapur dominieren Kooperationen auf nationaler und mondialer Ebene, während Kooperationspartnern im Integrationsraum ASEAN kaum Bedeutung zukommt. Nach Partnern differenziert, ergibt sich das gleiche Bild wie in den europäischen Untersuchungsregionen: Kooperationen mit Dienstleistern und Forschungseinrichtungen konzentrieren sich vornehmlich auf die regionale Ebene, während vertikale Verflechtungen am stärksten über die betrachteten Maßstabsebenen streuen. Die Unterschiede zwischen MNU und lokalen Unternehmen sind in Singapur eher graduell denn prinzipiell: Während MNU allgemein geringfügig stärker auf internationale Kooperationen ausgerichtet sind, arbeiten lokale Unternehmen aufgrund ihrer geringeren Größe häufiger mit Dienstleistern, aber seltener mit Forschungseinrichtungen zusammen.

Unter den in Penang befragten innovierenden Unternehmen sind Kooperationen weniger verbreitet als in Singapur. Die bestehenden innovationsrelevanten Verflechtungen sind in stärkerem Maße mondial ausgerichtet, da die technologische Basis in der Region weniger entwickelt ist als in Singapur. Letzteres ist auch für die Kooperationsreichweiten nach Partnern von Bedeutung: Anders als in ERIS-3 oder Singapur sind vertikale Kooperationen noch am stärksten lokalisiert, insbesondere downstream linkages mit Kunden. Anderswo stark regionalisierte Kooperationen mit Dienstleistern laufen in Penang v. a. auf der mondialen Ebene ab. Intensive Kooperationen mit Forschungseinrichtungen, die in der Frühphase des Innovationsprozesses bedeutsam und daher besonders auf räumliche Nähe angewiesen sind, haben in Penang dagegen praktisch keine Bedeutung. Diese Anomalien sind im Wesentlichen auf Angebotslücken in der technologischen Infrastruktur Penangs zurückzuführen.

Im Verlauf des Innovationsprozesses wird impli- 

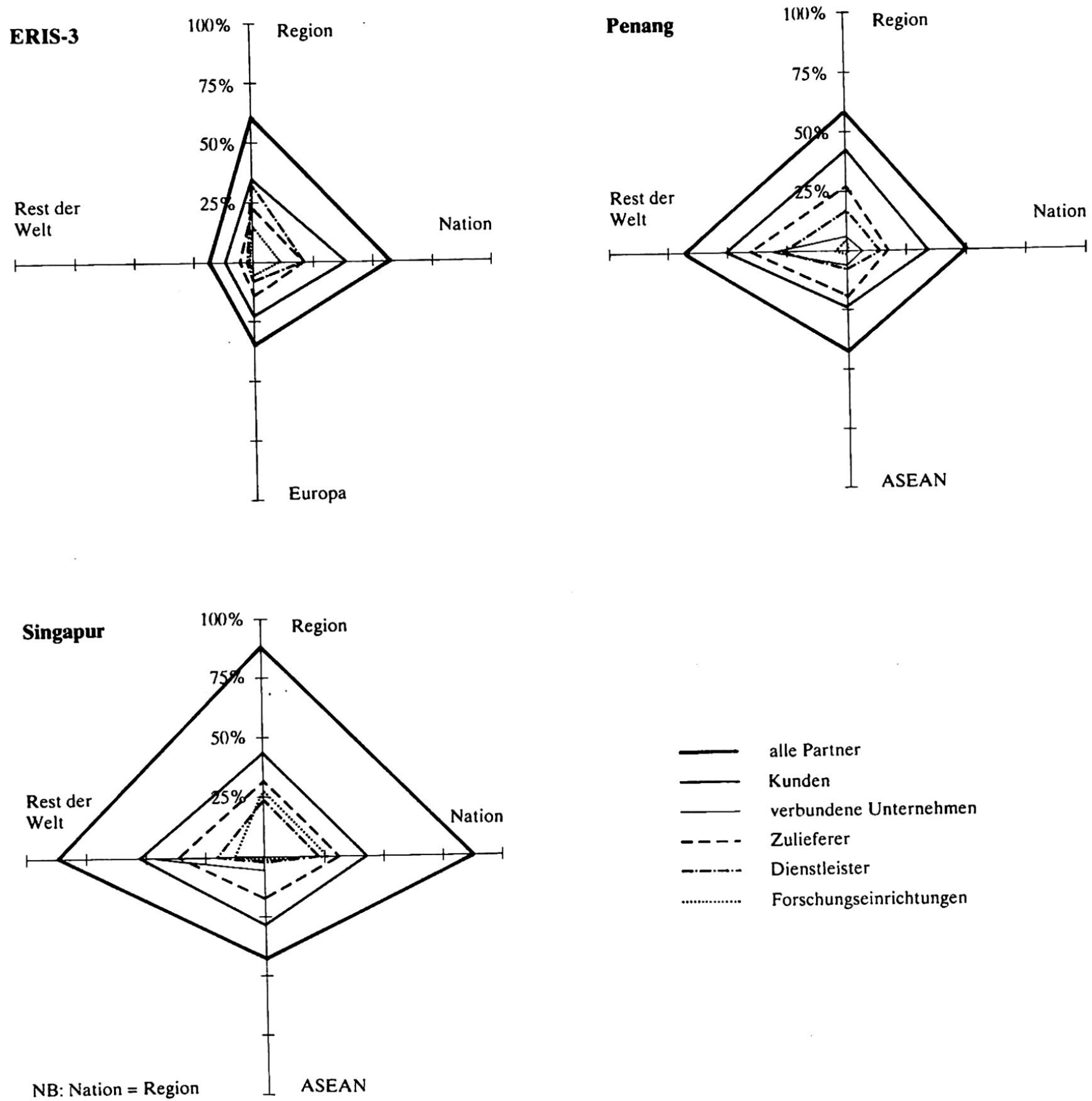

$100 \%$ = alle innovierenden Unternehmen

Abb. 1: Reichweiten intensiver innovationsrelevanter Kooperationsbeziehungen: Singapur und Penang im Vergleich mit den drei metropolitanen Innovationssystemen Barcelona, Stockholm und Wien (ERIS-3)

Spatial reach of intensive innovation-related linkages: Singapore and Penang in comparison with the metropolitan innovation systems of Barcelona, Stockholm and Vienna (ERIS-3)

Extension spatiale des relations intenses de coopération, en matière d'innovation marquante: Singapour et Penang, en comparaison avec les trois systèmes métropolitains d'innovation de Barcelone, Stockholm et Vienne (ERIS-3) Entwurf: M. KIESE; Graphik: V. SCHEURING 
zites Wissen kodifiziert und damit per Telekommunikation über beliebige räumliche Distanzen transportierbar. Daher ist zu erwarten, daß der räumlichen Nähe der Innovationsakteure zumindest in den Frühphasen des Prozesses eine große Bedeutung zukommt. Die in Südostasien vorgefundenen räumlichen Kooperationsmuster scheinen dieser Annahme zu widersprechen: Unternehmen kooperieren vor allem mit Partnern außerhalb Südostasiens. Die in Singapur durchgeführten Interviews liefern zwei Erklärungen, um diesen scheinbaren Widerspruch aufzulösen: Zum einen ist der Innovationsprozess räumlich aufgespalten, wobei die frühen Phasen des Innovationsprozesses zumeist außerhalb Singapurs in den Regionen der Kooperationspartner angesiedelt sind. Ideen für neue Produkte, Konzepte und auch Prototypen entstehen i.d.R. in den F\&E-Zentralen der MNU, wo auch deren Grundlagenforschung angesiedelt ist. Spätestens zur Pilotproduktion werden sie dann nach Singapur übertragen. Da die Technologie noch immer nicht vollständig konsolidiert und dokumentiert ist, erfordert der Transfer auch in dieser Phase noch face-to-face-Kontakte. Diese räumliche Nähe wird zum anderen künstlich hergestellt, indem die Unternehmen ihre Ingenieure zu meist mehrmonatigen Auslandsaufenthalten aussenden, um die neue Technologie zu erlernen. Nachfolgende Interaktionen und Rückkopplungen werden mit unterschiedlichen Mitteln der Telekommunikation (E-mail, Telefon, Fax, Videokonferenzen usw.) bewältigt.

Zusammenfassend belegen die erfaßten Innovationsund Kooperationsmuster die nach wie vor große Abhängigkeit der Untersuchungsregionen von externen Wissensquellen bzw. ihre noch gering entwikkelten endogenen Kapazitäten zur Produktion innovationsrelevanten Wissens. Unter den betrachteten Ländern bzw. Regionen ist Singapur bereits technologisch am weitesten entwickelt. Malaysias high-techEnklave Penang reicht bei den auf Unternehmensebene erhobenen Innovationsindikatoren bereits an Singapur heran, allerdings zeigen die Kooperationsmuster hier einen erheblichen Nachholbedarf im Aufbau einer innovationsrelevanten Infrastruktur (v.a. Forschungseinrichtungen und technologieorientierte Dienstleistungen) an.

\section{Fazit}

Die als Fortsetzung des European Regional Innovation Survey in Singapur, Penang und Thailand durchgeführten Innovationserhebungen erlauben es erstmals, regionale Innovationspotentiale und innovative Netzwerke in Südostasien nicht nur exemplarisch in Fallstudien darzustellen, sondern auf repräsentativer
Basis empirisch zu erfassen und vergleichend zu bewerten.

Sekundärstatistische Indikatoren weisen auf einen raschen technologischen Aufholprozess Singapurs hin, während sie für Malaysia und Thailand noch deutliche Rückstände in der technologischen Leistungsfähigkeit anzeigen. Die Erhebungsergebnisse auf Unternehmensebene belegen dagegen eine im Vergleich mit Europa noch immer deutlich geringere Verbreitung von Innovationsaktivitäten in Singapur, Penang und Thailand. Singapur hat bereits eine höhere technologische Entwicklungsstufe erreicht als Malaysia und Thailand. Innerhalb Malaysias steht die hightech-Enklave Penang jedoch auf einem ähnlichen technologischen Niveau wie Singapur, allerdings ist die technologische Infrastruktur der «Silicon Island» noch kaum entwickelt. Neben der allgemeinen «technologischen Lücke» läßt ein Vergleich der unterschiedlichen input-, throughput- und output-Indikatoren zudem auf eine geringere Effizienz betrieblicher Innovationsanstrengungen in Südostasien schließen.

Externe Kooperationen besitzen für innovierende Unternehmen in Singapur, Penang und Malaysia eine größere Bedeutung als in Europa, wichtigste Partner sind ausländische Mutterunternehmen für MNUTöchter sowie technologisch fortschrittliche Kunden (lead users) für lokale Unternehmen. Damit dominieren wie in Europa vertikale Kooperationsmuster. Während in Europa jedoch die Kooperationshäufigkeit mit zunehmender Entfernung der Partner abnimmt, herrscht in Singapur ein diskontinuierliches Raummuster vor. Unternehmen «überspringen» die technologisch weniger entwickelten Nachbarländer und kooperieren direkt mit technologisch führenden Partnern in Nordamerika, Europa und Japan. Räumliche Nähe ist dennoch von großer Bedeutung, wird jedoch bei der Übertragung von technischem Wissen im räumlich aufgespaltenen Innovationsproze $\beta$ durch Reisen der beteiligten Wissenschaftler und Ingenieure künstlich hergestellt.

Die Übertragung des in Europa erprobten Erhebungsinstrumentariums nach Südostasien ist im Bereich des Verarbeitenden Gewerbes als gelungen zu bezeichnen. Entscheidender Erfolgsfaktor war die ausdrückliche Unterstützung der Befragungen durch hochrangige politische Institutionen. Auf diesen Erfahrungen können zukünftige Innovationserhebungen im asiatischpazifischen Raum aufbauen. Neben der weiteren Verbreiterung der Vergleichsbasis durch Ausdehnung der bestehenden Methodik auf weitere Regionen erscheint jedoch auch der Aufbau eines Bestandes von Längsschnittdaten in ausgewählten Regionen sinnvoll, etwa im Rahmen von Folgebefragungen oder mit Hilfe einer Panelerhebung. 


\section{Literatur}

Agency for Science, Technology and Research (A*STAR) (2003): National Survey of R \& D in Singapore 2002. - Singapur: Agency for Science, Technology and Research.

Braczyk, H.J., Cooke, P. \& M. Heidenreich (Hrsg.) (1998): Regional Innovation Systems. The Role of Governance in a Globalised World. - London: Routledge.

COOKE, P. (1992): Regional Innovation Systems: Competitive Regulation in the New Europe. - In: Geoforum 23: 365-382.

CoOKe, P. \& K. Morgan (1998): The Associational Economy: Firms, Regions, and Innovation. - Oxford: Oxford University Press.

Chin, J. \& M.K. Lee (1999): Silicon Island. Penang Hones its High-tech Assets as it Races for 2010. - In: Asia Inc., September 1999: 28-31.

EUROPÄISCHE KOMMISSION (2001): Innovationsstatistik in Europa. Daten 1996-1997. - = Eurostat: Themenkreis 9, Wissenschaft und Technologie, Luxemburg: Amt für amtliche Veröffentlichungen der EG.

Freeman, C. (1987): Technology Policy and Economic Performance. Lessons from Japan. - London: Pinter.

FritsCh, M., KoschatZky, K., Schätzl, L. et al. (1998): Regionale Innovationspotentiale und innovative Netzwerke. - In: Raumforschung und Raumordnung 56, 4: 243-252.

GrabHer, G. (1993): Rediscovering the Social in the Economics of Interfirm Relations. - In: GrabHER, G. (ed.): The Embedded Firm. On the Socioeconomics of Industrial Networks. - London: Routledge: 1-31.

HÅKAnson, H. (1987): Product Development in Networks. - In: HÅKANSON, H. (Hrsg.): Industrial Technological Development. A Network Approach. - London: Croom Helm: 84-127.

HÅKANSON, H. \& I. SNEHOTA (1997): No Business is an Island. The Network Concept of Business Strategy. In: Scandinavian Journal of Management 4: 187-200.

HAYEK, F.A. vON (1976): Individualismus und wirtschaftliche Ordnung. - 2. Auflage, Salzburg: Neugebauer.

Herden, R. (1992): Technologieorientierte Außenbeziehungen im betrieblichen Innovationsmanagement: Ergebnisse einer empirischen Untersuchung. - = Wirtschaftswissenschaftliche Beiträge 65 , Heidelberg: Physica.

Hippel, E. von (1988): The Sources of Innovation. New York, Oxford: Oxford University Press.

Janz, N., Ebling, G., Gotrschalk, S. et al. (2001): The Mannheim Innovation Panels (MIP and MIP-S) of the Centre for European Economic Research (ZEW). In: Schmollers Jahrbuch, Zeitschrift für Wirtschaftsund Sozialwissenschaften 121: 123-129.

JANZ, N. \& G. Licht (Hrsg.) (1999): Innovationsaktivitäten in der deutschen Wirtschaft. Analyse der Mannheimer Innovationspanels im Verarbeitenden
Gewerbe und im Dienstleistungssektor. - = ZEWWirtschaftsanalysen 41, Baden-Baden: Nomos.

KIESE, M. (2004): Regionale Innovationspotentiale und innovative Netzwerke in Südostasien: Innovations- und Kooperationsverhalten von Industrieunternehmen in Singapur. - = Hannoversche Geographische Arbeiten 56, Münster, Hamburg: Lit Verlag.

Kline, S.J. \& N. Rosenberg (1986): An Overview of Innovation. - In: Landau, R. \& N. Rosenberg (eds): The Positive Sum Strategy: Harnessing Technology for Economic Growth. - Washington, DC: National Academy Press: 275-305.

Koschatzky, K. (2001): Räumliche Aspekte im Innovationsprozeß. Ein Beitrag zur neuen Wirtschaftsgeographie aus Sicht der regionalen Innovationsforschung. - = Wirtschaftsgeographie 19, Münster, Hamburg, London: LIT Verlag.

Krugman, P.R. (1994): The Myth of Asia's Miracle. In: Foreign Affairs 73, 4: 62-78.

Lundvall, B.-A. (ed.) (1992): National Systems of Innovation. Towards a Theory of Innovation and Interactive Learning. - London: Pinter.

Malaysian Science and Technology Information Centre (MASTIC) (2002): 2000 Survey of Research and Development. - Kuala Lumpur: Malaysian Science and Technology Information Centre.

Maskell, P., Eskelinen, H., Hannibalsson, I. et al. (1998): Competitiveness, Localised Learning and Regional Development. Specialization and Prosperity in Small Open Economies. - London, New York: Routledge.

MeYer, M. (1995): Die ökonomische Organisation der Industrie: Netzwerkarrangements zwischen Markt und Unternehmung. - = Neue betriebswirtschaftliche Forschung 140,Wiesbaden: Gabler.

Nelson, R.R. (ed.) (1993): National Systems of Innovation: A Comparative Study. - Oxford, New York, Toronto et al.: Oxford University Press.

ONG, C.I.A. (2001): Innovation in Penang's Manufacturing Sector. - Paper presented at the Regional Workshop on Innovation in the Manufacturing Sector, July 20, 2001, The Gurney Hotel, Penang.

Penrose, E.T. (1959): The Theory of the Growth of the Firm. - Oxford: Blackwell.

Picot, A. (1982): Der Transaktionskostenansatz in der Organisationstheorie: Stand der Diskussion und Aussagewert. - In: Die Betriebswirtschaft 42: 267-284. Polanyi, M. (1985): Implizites Wissen. - = Suhrkamp Taschenbuch Wissenschaft 453, Frankfurt a. M.: Suhrkamp.

Pralahad, C.K. \& G. Hamel (1990): The Core Competence of the Corporation. - In: Harvard Business Review 68, 3: 79-91.

Revilla Diez, J. (2000): The Importance of Public Research Institutions in Innovative Networks. Empirical Results from the Metropolitan Innovation Systems Barcelona, Stockholm and Vienna. - In: European Planning Studies 8, 4: 451-463. 
SCHÄTZL, L. (2000):Wirtschaftsgeographie 2. Empirie. 3. Auflage, Paderborn, München, Wien u.a.: Schöningh. SchäTZL, L. (2003): Wirtschaftsgeographie 1. Theorie. 9. Auflage, Paderborn, München, Wien u.a.: Schöningh. SchätZl, L. \& J. Revilla Diez (Hrsg.) (2002): Technological Change and Regional Development in Europe. - = Contribution to Economics, Heidelberg, New York: Springer.

SCHEIDT, B. (1995): Die Einbindung junger Technologieunternehmen in Unternehmens- und Politiknetzwerke. Eine theoretische, empirische und strukturpolitische Analyse. - = Volkswirtschaftliche Schriften 447, Berlin: Duncker \& Humblot.

Socio-EConomic and Environmental Research InSTITUTE (SERI) \& UNIVERSität HANNOVER (2001): Penang State Innovation Survey 2000. - Draft Internal Report. Penang, Hannover.

SternberG, R. (2000): Innovation Networks and Regional Development. Evidence from the European Regional Innovation Survey (ERIS): Theoretical Concepts, Methodological Approach, Empirical Basis and Introduction to the Theme Issue. - In: European Planning Studies 8, 4: 389-407.

Stracke, S. (2003): Technologische Leistungsfähigkeit im Innovationssystem Penang, Malaysia. - Dissertation, Geographisches Institut, Universität Hannover.

TöDTLING, F. (1994): The Uneven Landscape of Innovation Poles: Local Embeddedness and Global Networks. - In: Amin, A. \& N. Thrift (eds): Globalization, Institutions, and Regional Development. - Oxford u.a.: Oxford University Press: 68-90.

UNESCO (1999): Statistical Yearbook 1999. - Paris: UNESCO (United Nations Educational, Scientific and Cultural Organization).

VIRASA,T.\& P. BRIMBLE (2001): Technological Innovation of Industrial Enterprises in Thailand. - Paper presented at the Regional Workshop on Innovation in the Manufacturing Sector, July 20, 2001, The Gurney Hotel, Penang. http://www.seri.com.my/InnoWksp/ThailaPres.pdf, http://www.seri.com.my/InnoWksp/ThailaText.pdf (30.01.2002).

Weltbank (1993): The East Asian Miracle: Economic Growth and Public Policy. - Oxford, New York, Toronto u.a.: Oxford University Press.

Williamson, O.E. (1989): Transaction Cost Economics. - In: Schmalensee, R. \& R.D.Willig (eds): Handbook of Industrial Organization 1. - = Handbook in Economics 10,1, Amsterdam u.a.: North Holland: 135-182.

Wong, P.K. (1995): National Innovation System: The Case of Singapore. - Seoul: STEPI (Science and Technology Policy Institute).

WoNG, P.K. (1999): National Innovation Systems for Rapid Technological Catch-up: An Analytical Framework and a Comparative Analysis of Korea, Taiwan and Singapore. - Paper presented at the Danish Research Unit for Industrial Dynamics (DRUID),
Summer Conference on National Innovation Systems, Industrial Dynamics and Innovation Policy, Rebild (Denmark), June 9-12, 1999.

Wong, P.K., Kiese, M., Singh, A. et al. (2003): The Pattern of Innovation in Singapore's Manufacturing Sector. - In: Singapore Management Review 25, 1: 1-34.

\section{Zusammenfassung: Regionale Innovationspotentiale in Südostasien: Empirische Ergebnisse aus Singapur, Penang (Malaysia) und Thailand}

Aufbauend auf den Erfahrungen des European Regional Innovation Survey (ERIS) versucht der vorliegende Beitrag erstmals, das regionale Innovationspotential sowie die Kooperationsbeziehungen zwischen den Innovationsakteuren in ausgewählte Regionen Südostasiens empirisch zu erfassen und vergleichend zu bewerten. Repräsentative Befragungen in Singapur, Penang (Malaysia) und Thailand ergaben verwertbare Informationen zum Innovations- und Kooperationsverhalten von rund 1.600 Unternehmen des Verarbeitenden Gewerbes. Die Ergebnisse zeigen, daß die Breite und Effizienz der Innovationsaktivitäten in den Untersuchungsregionen noch nicht mit der Situation europäischer Regionen vergleichbar sind. Kooperationen sind für die Innovationsprozesse der Unternehmen praktisch unverzichtbar; die wichtigsten Partner finden sich für multinationale Unternehmen im Konzernverbund, während einheimische Unternehmen meist mit Kunden (lead users) in hochentwickelten Technologieregionen kooperieren. Hieraus resultiert ein diskontinuierliches Raummuster, in dem die benachbarten Länder und Regionen Südostasiens übersprungen werden.

\section{Summary: Regional Innovative Potential in Southeast Asia: Empirical evidence from Singapore, Penang (Malaysia) and Thailand}

Based on experiences from the European Regional Innovation Survey (ERIS), this paper reports on a first attempt to measure and compare regional innovative potential and innovation-related co-operations in selected parts of Southeast Asia. Around 1.600 manufacturing firms responded to our representative surveys in Singapore, Penang (Malaysia) and Thailand. Our results show that despite remarkable catching-up in Singapore, the breadth and efficiency of innovative activities still lags considerably behind that found in eleven European regions. Co-operation is virtually indispensable for corporate innovation processes in the region. Subsidiaries of multinational corporations are most likely to collaborate with their corporate headquarters, or R \& D centres respectively. In contrast, lead users in technologically advanced countries are the prime sources of knowledge for endogenous firms. Both leads to a discontinuous spatial pattern of 
collaboration linkages in which companies «leapfrog» neighbouring regions and countries to work with partners further afield.

\section{Résumé: Potentiels d'innovation régionaux dans le Sud-Est asiatique: résultats empiriques relatifs à Singapour, à Penang (Malaisie) et à la Thailande}

La présente contribution essaie pour la première fois de cerner empiriquement et de manière comparée le potentiel régional d'innovation, ainsi que les rapports de coopération entre les acteurs de l'innovation, à partir d'un choix de régions du Sud-Est asiatique, s'appuyant en cela sur l'expérience acquise dans le cadre de l'«European Regional Innovation Survey» (ERIS). Des investigations représentatives réalisées à Singapour, à Penang (Malaisie) et en Thaïlande ont fourni des informations utilisables pour l'étude du comportement dans le domaine de l'innovation et de la coopération, d'environ 1600 entreprises des activités de transformation. Les résultats obtenus révèlent que ni l'extension spatiale ni l'efficacité des activités portant sur l'innovation dans les régions concernées par l'enquête ne sont déjà comparables avec la situation existante dans les régions européennes. Les coopérations portant sur les processus d'innovation des entreprises sont pratiquement incontournables; les partenaires les plus importants, pour ce qui est des firmes multinationales, sont intégrés au groupe, tandis que des entreprises autochtones coopèrent la plupart du temps avec des clients (lead users) localisés dans des régions déjà avancées dans les hautes technologies. Il en résulte un modèle spatial discontinu, en «saut de mouton» par rapport aux Etats et régions voisins du Sud-Est asiatique.

\section{Didaktische Hinweise}

- Welche Ursachen sind für den wirtschaftlichen Erfolg Südostasiens verantwortlich?

- Warum ist eine zunehmende technologische Leistungsfähigkeit für eine nachhaltige Wirtschaftsentwicklung der Staaten Südostasiens notwendig?

- Welche Rolle spielt in der theoretischen Diskussion das Vorhandensein geeigneter Kooperationspartner bei betrieblichen Innovationsprozessen?
- Schlagen sich die neuen Erkenntnisse der Innovationsforschung auch in den vorgestellten Regionalstudien nieder?

- Lassen sich Unterschiede im Kooperationsverhalten zwischen europäischen und südostasiatischen Unternehmen erkennen?

- Welche Bedeutung hat die räumliche Nähe zwischen Kooperationspartnern bei betrieblichen Innovationsprozessen aus theoretischer Sicht?

- Lassen sich Unterschiede in den räumlichen Reichweiten der Kooperationspartner in den vorgestellten Regionalstudien feststellen?

- Welche Rolle spielt das lokale Umfeld bei der Entwicklung neuer Produkte und Verfahren?

Dipl.-Geogr. Matthias Kiese, Geographisches Institut der Universität Hannover, Schneiderberg 50, D-30167 Hannover.

e-mail: kiese@wigeo.uni-hannover.de

Prof. Dr. Javier Revilla Diez, Geographisches Institut der Christian-Albrechts-Universität zu Kiel, Lehrstuhl für Wirtschaftsgeographie, Ludewig-Meyn-Strasse 14, D-24098 Kiel.

e-mail:diez@geographie.uni-kiel.de

\section{Manuskripteingang/received/manuscrit entré le} 27.2.2002

Annahme zum Druck/accepted for publication/accepté pour l'impression: 16.2.2004 